\title{
Quantum information dynamics in a high-dimensional parity-time-symmetric system
}

\author{
Zhihao Bian, ${ }^{1,2}$ Lei Xiao, ${ }^{1}$ Kunkun Wang, ${ }^{1}$ Franck Assogba Onanga, ${ }^{3}$ Frantisek Ruzicka, ${ }^{3,4}$ Wei Yi, ${ }^{5,6, *}$ \\ Yogesh N. Joglekar $\left({ }^{3},{ }^{3, \dagger}\right.$ and Peng Xue (1) ${ }^{1, \dagger}$ \\ ${ }^{1}$ Beijing Computational Science Research Center, Beijing 100084, China \\ ${ }^{2}$ School of Science, Jiangnan University, Wuxi 214122, China \\ ${ }^{3}$ Department of Physics, Indiana University Purdue University Indianapolis (IUPUI), Indianapolis, Indiana 46202, USA \\ ${ }^{4}$ Institute of Nuclear Physics, Czech Academy of Sciences, Rez 250 68, Czech Republic \\ ${ }^{5}$ CAS Key Laboratory of Quantum Information, University of Science and Technology of China, Hefei 230026, China \\ ${ }^{6}$ CAS Center For Excellence in Quantum Information and Quantum Physics, Hefei 230026, China
}

(Received 30 April 2020; accepted 18 August 2020; published 16 September 2020)

\begin{abstract}
Non-Hermitian systems with parity-time $(\mathcal{P} \mathcal{T})$ symmetry give rise to exceptional points (EPs) with exceptional properties that arise due to the coalescence of eigenvectors. Such systems have been extensively explored in the classical domain, where second- or higher-order EPs have been proposed or realized. In contrast, quantum information studies of $\mathcal{P} \mathcal{T}$-symmetric systems have been confined to systems with a two-dimensional Hilbert space. Here, by using a single-photon interferometry setup, we simulate the quantum dynamics of a four-dimensional $\mathcal{P} \mathcal{T}$-symmetric system across a fourth-order exceptional point. By tracking the coherent, nonunitary evolution of the density matrix of the system in $\mathcal{P} \mathcal{T}$-symmetry unbroken and broken regions, we observe the entropy dynamics for both the entire system, and the gain and loss subsystems. Our setup is scalable to the higher-dimensional $\mathcal{P} \mathcal{T}$-symmetric systems, and our results point towards the rich dynamics and critical properties.
\end{abstract}

DOI: 10.1103/PhysRevA.102.030201

\section{INTRODUCTION}

A fundamental postulate of quantum theory is that the Hamiltonian of an isolated system is Hermitian. This Hermiticity ensures real eigenvalues and a coherent, unitary time evolution for the system. This conventional wisdom was upended two decades ago by Bender and co-workers, who showed that a non-Hermitian Hamiltonian with parity-time $(\mathcal{P} \mathcal{T})$ symmetry can exhibit entirely real spectra [1-4]. Over time, it has become clear that non-Hermitian Hamiltonians with $\mathcal{P} \mathcal{T}$ symmetry can provide an effective description for systems with balanced, spatially separated gain and loss [5]. This concept has been extensively, and fruitfully, explored in classical (wave) systems where the number of energy quanta is much larger than one [6-15]. A $\mathcal{P} \mathcal{T}$-symmetric system is described by an effective, non-Hermitian Hamiltonian $H_{\mathcal{P} \mathcal{T}}$ that is invariant under the combined parity and time-reversal operation [16]. As the gain-loss strength is increased, the spectrum of $H_{\mathcal{P}} \mathcal{T}$ changes from real into complex conjugate pairs, and the corresponding eigenvectors cease to be eigenvectors of the $\mathcal{P} \mathcal{T}$ operator. This $\mathcal{P} \mathcal{T}$-symmetry-breaking transition occurs at an EP of order $n(\mathrm{EP} n)$, where $n$ eigenvalues, as well as their corresponding eigenvectors, coalesce [17-19]. The $\mathcal{P} \mathcal{T}$ transition and the nonunitary time evolution generated by $H_{\mathcal{P} \mathcal{T}}$ have been observed in classical systems

\footnotetext{
*wyiz@ustc.edu.cn

$\dagger$ yojoglek@iupui.edu

‡gnep.eux@gmail.com
}

with EP2 [6-14,20-26], EP3 [15], and higher-order EPs $[27,28]$.

Due to the quantum limit on noise in linear (gain) amplifiers [29], creating a photonic system with balanced gain and loss in the quantum domain is not possible [30]. However, the EP degeneracies also occur in dissipative systems with mode-selective losses. Such passive $\mathcal{P} \mathcal{T}$-symmetric systems have been realized in the quantum domain with lossy, single photons [31-38], ultracold atoms [39], and a superconducting transmon [40]. These realizations are limited to effective two-dimensional Hamiltonians with second-order EPs, and their quantum information studies are confined to global properties [34]. Here, we present the experimental quantum simulation of entropy dynamics in a four-dimensional, passive $\mathcal{P} \mathcal{T}$-symmetric system with an EP4.

\section{IMPLEMENTING $\mathcal{P} \mathcal{T}$-SYMMETRIC QUDIT WITH AN EP4}

Let us consider an open, four-mode system described by a $4 \times 4$ Hamiltonian

$$
H_{\mathcal{P} \mathcal{T}}=-J S_{x}+i \gamma S_{z}
$$

where $S_{x}$ and $S_{z}$ are spin-3/2 representations of the SU(2) group. It can be written in the matrix form as

$$
H_{\mathcal{P} \mathcal{T}}=\frac{1}{2}\left(\begin{array}{cccc}
3 i \gamma & -\sqrt{3} J & 0 & 0 \\
-\sqrt{3} J & i \gamma & -2 J & 0 \\
0 & -2 J & -i \gamma & -\sqrt{3} J \\
0 & 0 & -\sqrt{3} J & -3 i \gamma
\end{array}\right)
$$



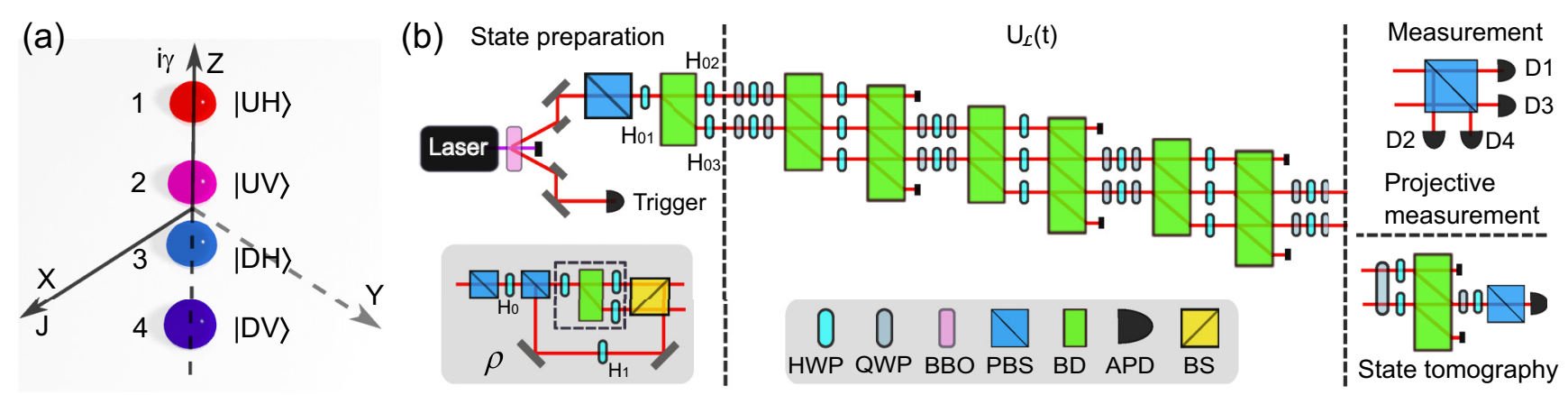

FIG. 1. Experimental setup. (a) Illustration of a four-mode $\mathcal{P} \mathcal{T}$-symmetric qudit. (b) Schematic of the optical circuit used for simulating dynamics in the four-mode, passive $\mathcal{P} \mathcal{T}$-symmetric system. Heralded single photons are generated via spontaneous parametric down-conversion and prepared in arbitrary qudit states using a polarizing beam splitter (PBS), wave plates with certain setting angles, and a beam displacer (BD). The coherent, lossy, nonunitary time evolution is realized by BDs, HWPs, and sandwich-type QWP-HWP-QWP setups, along with single-photon loss. For detection, projective measurements and quantum-state tomography are selected depending on the purpose, either of which is performed, both of which are realized by a PBS and (or) wave plates and a BD. Avalanche photodiodes (APDs) detect the signal and heralding photons.

in the computational basis $\{|1\rangle,|2\rangle,|3\rangle,|4\rangle\}$, and represents a $\mathcal{P} \mathcal{T}$-symmetric qudit with $d=4$. The Hamiltonian $H_{\mathcal{P} \mathcal{T}}$ commutes with the antilinear $\mathcal{P} \mathcal{T}$ operator where the parity operator is $\mathcal{P}=$ antidiag $(1,1,1,1)$ and a time-reversal operator is given by complex conjugation, $\mathcal{T}=*$. It follows from Eq. (2) that the first two computational modes represent the "gain sector" and the last two represent the "loss sector" in the system. The four equally spaced eigenvalues of $H_{\mathcal{P} \mathcal{T}}$ are given by $\lambda_{k}=\{-3 / 2,-1 / 2,+1 / 2,+3 / 2\} \sqrt{J^{2}-\gamma^{2}}(k=$ $1,2,3,4)$, which give rise to an EP4 at the $\mathcal{P} \mathcal{T}$-breaking threshold $\gamma=J$. The advantage of choosing Hamiltonian (1) is that it can be easily generalized to an arbitrary dimensional system where it still remains analytically solvable and has an EP with the order equal to the system dimension $[15,41,42]$. Since $H_{\mathcal{P} \mathcal{T}}$ has a single energy gap $\Delta=\sqrt{J^{2}-\gamma^{2}}$, it follows that the $\mathcal{P} \mathcal{T}$-symmetric qudit has a sinusoidal dynamics in the $\mathcal{P} \mathcal{T}$-symmetry unbroken region $(\gamma<J)$, and a monotonic, exponential growth behavior in the $\mathcal{P} \mathcal{T}$-broken region $(\gamma>J)$.

The coherent, nonunitary time evolution operator for the system is given by $U(t)=\exp \left(-i H_{\mathcal{P} \mathcal{T}} t\right)$, where we have set $\hbar=1$. For $\gamma=0$, the system is Hermitian and the fermionic nature of the spin-3/2 representation is manifest in the antiperiodicity of $U$, i.e., $U(T)=-\mathbb{I}_{4}$, where $T(0)=2 \pi / J$ for $\gamma=0$. In this case, the mode occupations $P_{k}(t)=|\langle k \mid \psi(t)\rangle|^{2}$ of the four modes obey a shifted mirror symmetry with $P_{k}(t)=P_{5-k}(t+T / 2)$, which indicates a perfect state transfer occurring from mode $k$ to mode $(5-k)$ at $T / 2$. Here, $|\psi(t)\rangle=U(t)|\psi(0)\rangle$ is the time-evolved state. For $\gamma<J$, the system is in the $\mathcal{P} \mathcal{T}$-symmetry unbroken region, and the dynamical evolution is antiperiodical with period $T(\gamma)=$ $2 \pi / \Delta$. At the EP4 $(\gamma=J), U(t)$ ceases to be periodic and has an operator norm that grows as $t^{6}$, reflecting the fourth order of the EP. In the $\mathcal{P} \mathcal{T}$-symmetry broken region, the mode occupations grow exponentially with time. However, the quantum information metrics, such as the von Neumann entropy, are defined with respect to the instantaneously normalized state (indicating postselection that eliminates the quantum jumps $[38,40,42])$. Therefore, at the EP and in the $\mathcal{P} \mathcal{T}$-broken region, these quantities reach a steady-state value.
These results are applicable to all finite-dimensional representation of the SU(2) group.

The four-dimensional Hamiltonian $H_{\mathcal{P} \mathcal{T}}$ is particularly interesting because it can be viewed as a system of two interacting, non-Hermitian qubits. This mapping is provided by the identities $2 S_{x}=\sigma_{x} \otimes \sigma_{x}+\sigma_{y} \otimes \sigma_{y}+\sqrt{3} \mathbb{I}_{2} \otimes \sigma_{x}, 2 S_{z}=$ $\sigma_{z} \otimes \mathbb{I}_{2}+\mathbb{I}_{2} \otimes \sigma_{z} / 2$, and $\mathcal{P}=\sigma_{x} \otimes \sigma_{x}$, where $\sigma_{k}(k=x, y, z)$ are the standard Pauli matrices. Using this insight, we investigate the quantum information dynamics in the gain and loss subsystems of the $\mathcal{P} \mathcal{T}$-symmetric qudit.

We encode the four modes of the qudit in the spatial and polarization degrees of freedom of a single photon, and label them as $|1\rangle=|U H\rangle,|2\rangle=|U V\rangle,|3\rangle=|D H\rangle,|4\rangle=$ $|D V\rangle$. Here, $\{|H\rangle,|V\rangle\}$ are the horizontal and vertical polarizations, and $\{|U\rangle,|D\rangle\}$ denote the upper and lower paths, which undergo gain and loss, respectively [Fig. 1(a)]. As illustrated in Fig. 1(b), pairs of single photons are generated via type-I spontaneous parametric down-conversion (SPDC) using a nonlinear $\beta$-barium-borate (BBO) crystal. One photon serves as a trigger and the other signal photon is prepared in an arbitrary qudit state using a polarizing beam splitter (PBS), wave plates with certain setting angles, and a beam displacer (BD).

By mapping the $\mathcal{P} \mathcal{T}$-symmetric Hamiltonian $H_{\mathcal{P} \mathcal{T}}$ into a passive $\mathcal{P} \mathcal{T}$-symmetric one with mode-selective losses $H_{\mathcal{L}}=H_{\mathcal{P} \mathcal{T}}-3 i \gamma \mathbb{I}_{4} / 2$, we implement the $4 \times 4$ lossy, timeevolution operator

$$
U_{\mathcal{L}}(t)=\exp \left(-i H_{\mathcal{L}} t\right)
$$

via a lossy linear optical circuit, which is related to $U(t)$ through $U(t)=U_{\mathcal{L}}(t) \exp (3 \gamma t / 2)$ [31]. The evolution operator $U_{\mathcal{L}}(t)$ is realized by BDs, half-wave plates (HWPs), and sandwich-type QWP-HWP-QWP setups, where QWP is an abbreviation for quarter-wave plate.

We experimentally measure and then obtain scaled mode occupations $P_{k}(t)$ by projecting the time-evolved state $|\psi(t)\rangle$ onto $|k\rangle$. The initial state is chosen to be $|\psi(0)\rangle=(|1\rangle+$ $|2\rangle+|3\rangle+|4\rangle) / 2$. The projective measurement and the quantum state tomography on the qudit state are realized by BDs, wave plates, and a PBS followed by avalanche photodiodes 

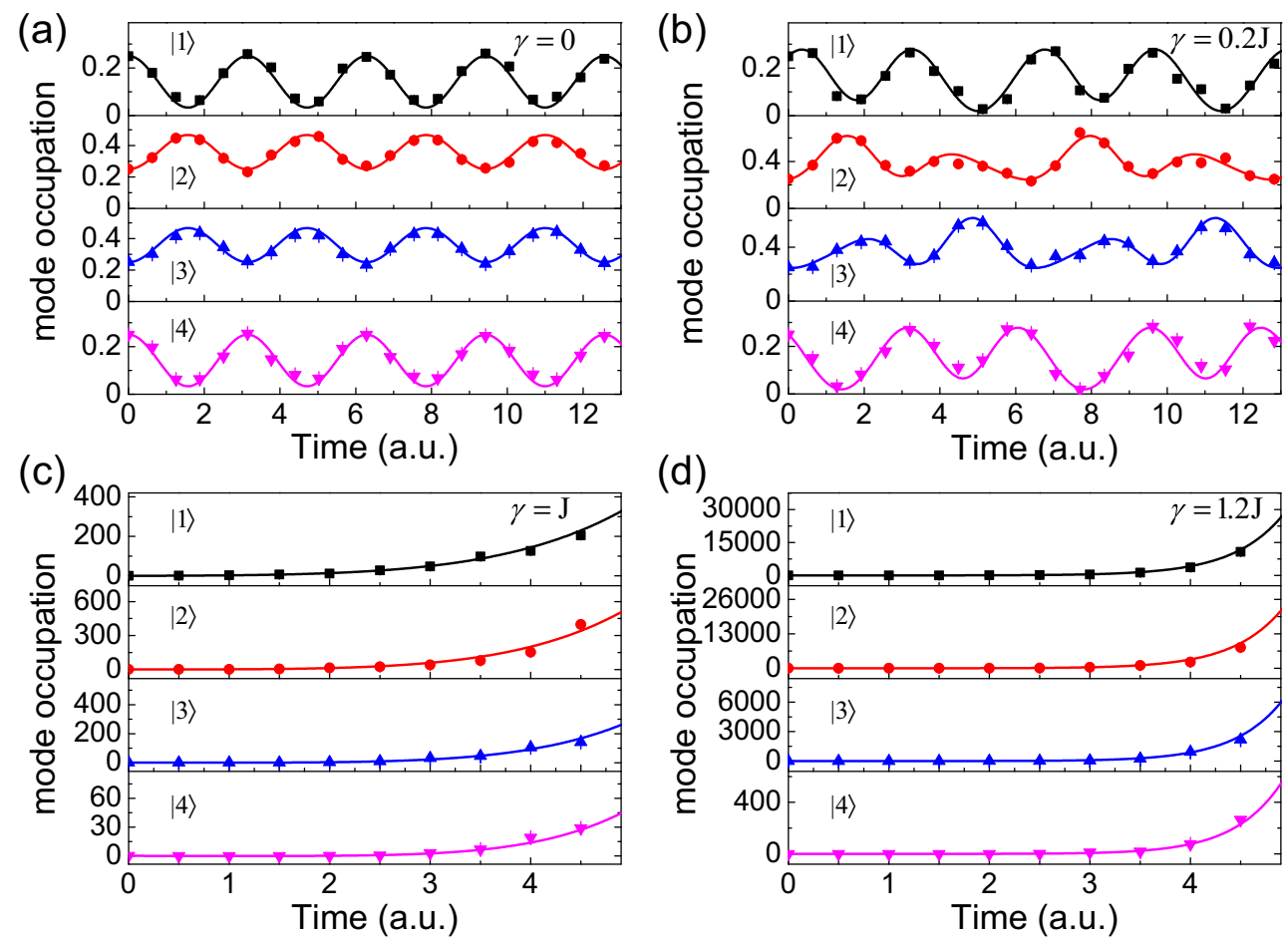

(d)

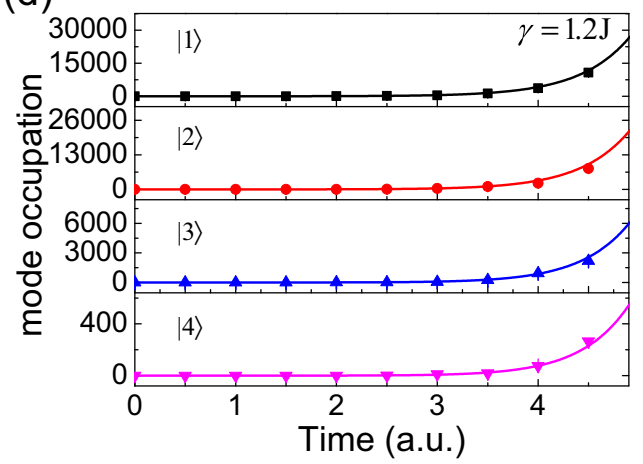

FIG. 2. Scaled mode occupations of a $\mathcal{P} \mathcal{T}$-symmetric system. (a) In the Hermitian limit with $\gamma=0$, mode occupation numbers are periodic with period $T=2 \pi / J$, and perfect state transfer occurs from mode $k$ to mode $5-k$ at time $T / 2$. (b) In the $\mathcal{P} \mathcal{T}$-unbroken phase with $\gamma=0.2 J$, the occupation dynamics have period $T(\gamma)$, and the total weights exceed unity indicating a non-trace-preserving time evolution. (c) At $\gamma=J$, the scaled mode occupation grows as $t^{6}$ with time indicating that the exceptional point is of order four. (d) In the $\mathcal{P} \mathcal{T}$-broken phase with $\gamma=1.2 J$, the scaled mode occupation grows exponentially with time. Time is measured in units of $J$. Symbols: data; lines: Theory. Error bars are due to the statistical uncertainty and obtained based on assuming Poisson statistics. When not shown, error bars are smaller than the symbol size.

(APDs). Only coincidences between the heralded and trigger photons are registered. The perfect state transfer for $\gamma=0$ is confirmed by the transfer of occupation from the first mode to the fourth mode [Fig. 2(a)]. In the $\mathcal{P} \mathcal{T}$-unbroken phase with a finite $\gamma=0.2 \mathrm{~J}$, there is no perfect state transfer at time $T(\gamma) / 2$ due to the nonunitary dynamics [Fig. 2(b)]. The measured occupations are, however, periodic in time with a period $T(\gamma)$.

At the EP4 with $\gamma=J$, the scaled mode occupation $P_{k}(t)$ grows algebraically with time as $t^{6}$ [Fig. 2(c)]. Such a scaling is dictated by the order of the EP. At $\gamma=J$, the Hamiltonian obeys $H_{\mathcal{P} \mathcal{T}}^{4}(\gamma=J)=0$ and the power-series expansion of $U(t)$ terminates at the third order, giving rise to $t^{6}$ dependence for the occupation numbers. By projecting the time-evolved state onto $|k\rangle$, we can obtain the occupation at the EP4 and its power-law behavior [Fig. 2(c)]. In the $\mathcal{P} \mathcal{T}$-broken phase, the scaled mode occupation grows exponentially with time as expected [Fig. 2(d)]. We note that while the simulation time range is limited to two periods for $\gamma<J$, we restrict to $0 \leqslant t \leqslant 4.5$ due to the rapid growth of the scaled mode occupation at the EP4 and in the broken $\mathcal{P} \mathcal{T}$ region.

When the $\mathcal{P} \mathcal{T}$-symmetric Hamiltonian is perturbed from the EP4 by a small detuning $\delta$, the resulting complex eigenvalues in the vicinity of EPn are given by a Puiseux series in $\delta^{1 / n}$ [41], indicating enhanced classical sensitivity proportional to the order of the EP $[15,43]$. In addition to the behavior of the mode occupations at the EP, this serves as a complementary check of the order of the EP. To that end, we experimentally measure the complex eigenvalues of the perturbed Hamiltonian $H_{\delta}=H_{\mathcal{P} \mathcal{T}}(\gamma=J)+i J \delta|1\rangle\langle 1|$. Figure 3 shows that the real and imaginary parts of the eigenvalues of $H_{\delta}$ indeed scale as $\delta^{1 / 4}$, consistent with the EP4 that occurs at $\gamma=J$.

\section{OBSERVING INFORMATION DYNAMICS}

A crucial aspect of dynamics of a high-dimensional $\mathcal{P} \mathcal{T}$ symmetric system is the flow of information among its different parts, and the information retrieval phenomena between the whole system and its environment. To that end, we consider the qudit entropy

$$
S(t)=-\operatorname{Tr}\left[\tilde{\rho}(t) \log _{2} \tilde{\rho}(t)\right],
$$

where $\tilde{\rho}(t)=\rho(t) / \operatorname{Tr}[\rho(t)]$ is the instantaneously normalized density matrix and $\rho(t)=U(t) \rho(0) U^{\dagger}(t)$ is the time-evolved density matrix of the system with a time-dependent trace. The gain- and loss-sector entropies are $S_{\mathrm{Gain}}(t)$ and $S_{\mathrm{Loss}}(t)$, respectively. These are obtained from the gain- and loss-sector reduced density matrices $\rho_{\text {Gain }}(t)=\operatorname{Tr}_{3,4}[\rho(t)]$ and $\rho_{\text {Loss }}(t)=$ $\operatorname{Tr}_{1,2}[\rho(t)]$, respectively.

A full knowledge of the time-dependent density matrix through the quantum-state tomography allows us to experimentally explore the information flow. We focus on the quantum dynamics with the fully symmetric 

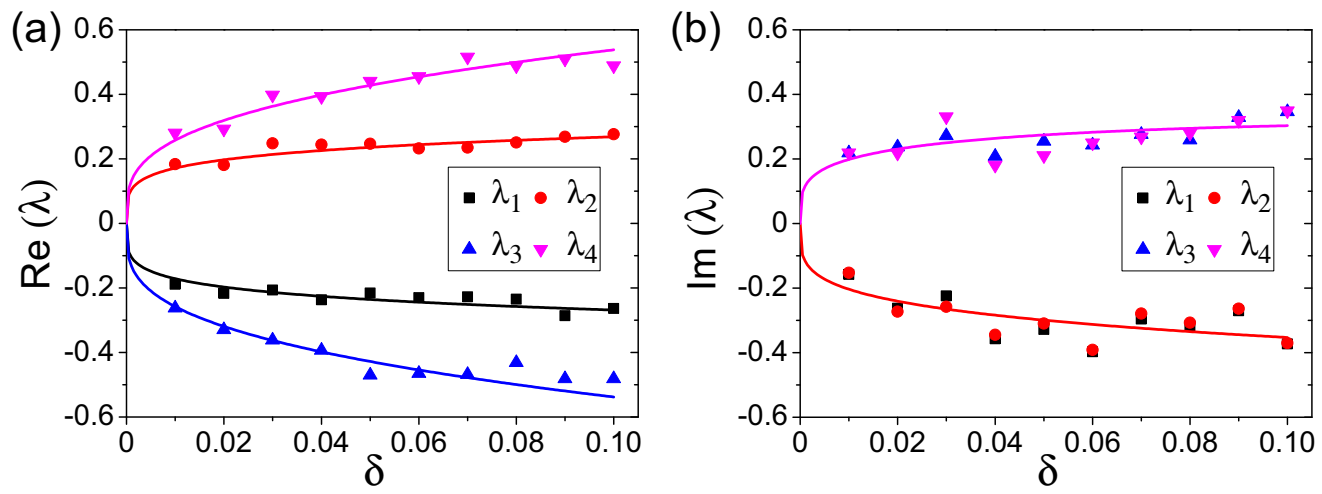

FIG. 3. Eigenvalues of the perturbed Hamiltonian. (a) The real and (b) imaginary parts of the eigenvalues of the perturbed Hamiltonian $H_{\delta}=H_{\mathcal{P} \mathcal{T}}(\gamma=J)+i J \delta|1\rangle\langle 1|$, measured in units of $J$, scale as $\delta^{1 / 4}$, showing the enhanced sensitivity near the EP4. The initial states are eigenstates $\left|\psi_{\delta}\right\rangle$ of $H_{\delta}$. Experimental errors are calculated via Monte Carlo method; when not shown, error bars are smaller than the symbol size.

initial state $|\psi(0)\rangle$ [Figs. 4(a)-4(c)] and a mixed initial state $\quad \rho(0)=0.925|1\rangle\langle 1|+0.025(|2\rangle\langle 2|+| 3\rangle\langle 3|+| 4\rangle\langle 4|)$ [Figs. 4(d)-4(f)] in the $\mathcal{P} \mathcal{T}$-symmetry unbroken region. Since the qudit undergoes a coherent, nonunitary evolution for any gain-loss strength $\gamma$, a pure state remains a pure state and the entropy of the entire system $S(t)$ remains constant with time [Fig. 4(a)]. For a mixed initial state, the entropy is constant only in the Hermitian limit, $\gamma=0$. In the $\mathcal{P} \mathcal{T}$-symmetry unbroken region, the entropy $S(t)$ shows periodic oscillations. This demonstrates an exchange of quantum information between the $\mathcal{P} \mathcal{T}$-symmetric qudit and its environment, and the oscillations observed here may be interpreted as evidence of information backflow from the environment and a signature of non-Markovianity in the $\mathcal{P} \mathcal{T}$-unbroken phase [34].

At the EP4 or in the $\mathcal{P} \mathcal{T}$-symmetry broken region, due to the diverging occupation, the normalized density matrix

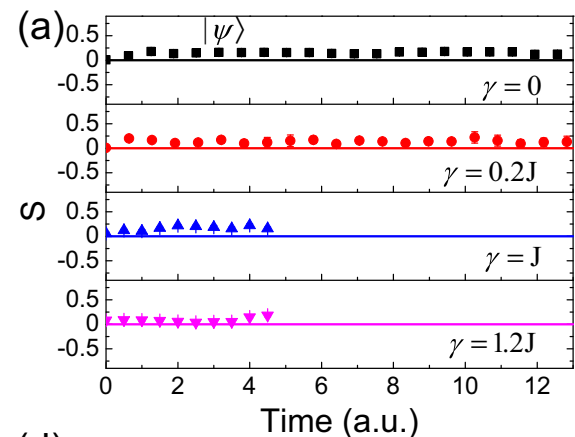

(d)

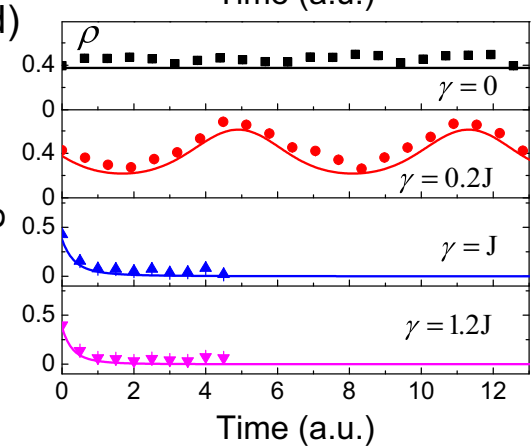

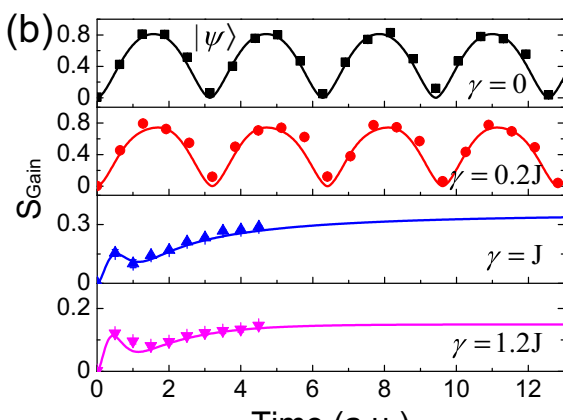

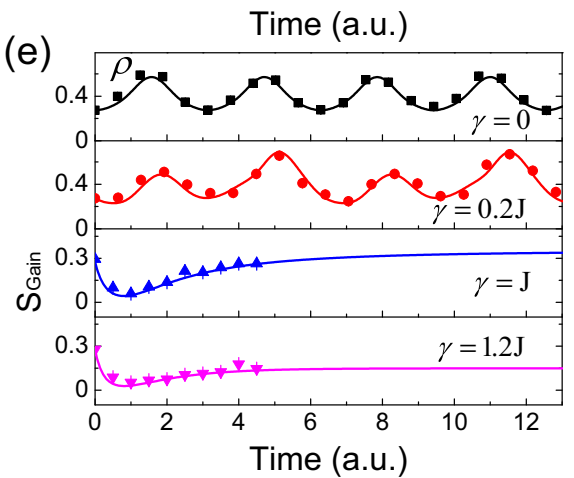

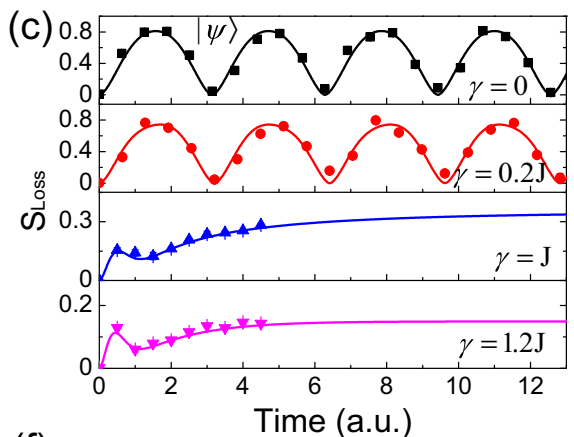

(f)

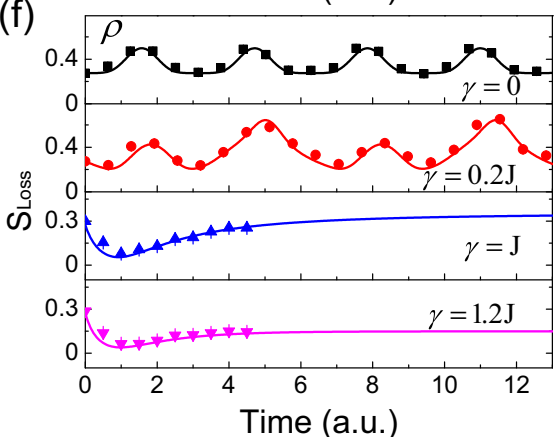

FIG. 4. Quantum information dynamics of the $\mathcal{P} \mathcal{T}$-symmetric qudit. (a) Time-dependent entropy $S(t)$ for a qudit with a pure initial state $|\psi(0)\rangle$ remains zero for any non-Hermiticity. (d) With a mixed initial state, $S(t)$ is constant when $\gamma=0$, and oscillates in the $\mathcal{P} \mathcal{T}$-unbroken phase $(\gamma=0.2 J)$ with period $T(\gamma)$. At the EP4 $(\gamma=J)$ and in the broken $\mathcal{P} \mathcal{T}$ region, $S(t)$ reaches zero because the system approaches a pure state that is determined by the sole eigenstate at the EP or the mode with maximum amplification. In contrast, the subsystem entropies $S_{\text {Gain }}(t)$ and $S_{\text {Loss }}(t)$ show qualitatively similar behavior for (b), (c) pure and (e), (f) mixed initial qudit states. In each case, the entropies show oscillatory behavior for $\gamma<J$ and steady-state behavior for $\gamma \geqslant J$. We restrict to $0 \leqslant t \leqslant 4.5$ due to the rapid growth of the scaled mode occupation at the EP4 and in the broken $\mathcal{P} \mathcal{T}$ region. Experimental errors are due to Monte Carlo method; when not shown, error bars are smaller than the symbol size. 

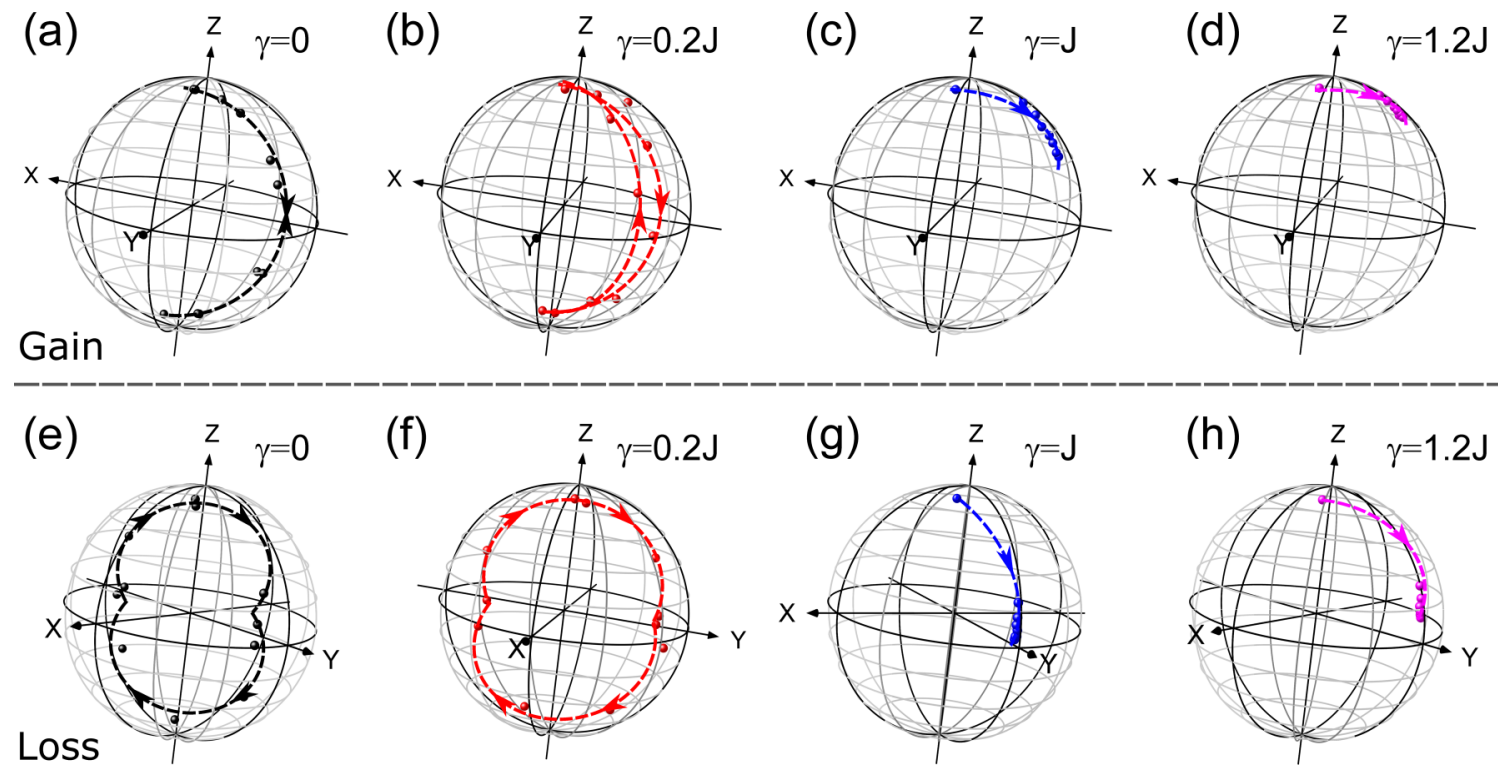

FIG. 5. Reduced, instantaneously normalized density matrices (a)-(d) $\tilde{\rho}_{\text {Gain }}(t)$ and (e)-(h) $\tilde{\rho}_{\text {Loss }}(t)$ trace different paths inside the Bloch sphere. Experimental results are represented by colored symbols and their theoretical predictions are represented by dashed curves.

$\tilde{\rho}(t)$ approaches a pure state, and the total system entropy therefore approaches zero [34,44,45]. In all cases, the experimental simulation results agree well with the theoretical prediction. Importantly, this observed behavior of entropy does not depend on the details of the system, which signifies its universality. In this case, information flows unidirectionally and the dynamics is asymptotically Markovian [44].

In a sharp contrast to the results for the entire system, the behavior of subsystem entropies for pure and mixed initial states is qualitatively similar. In either case, the gain-sector entropy $S_{\text {Gain }}(t)$ and the loss-sector entropy $S_{\text {Loss }}(t)$ oscillate in the $\mathcal{P} \mathcal{T}$-symmetry unbroken region including the Hermitian limit. On the other hand, they reach nonzero steady-state values at EP4 and in the broken $\mathcal{P} \mathcal{T}$-symmetry region. It is worthwhile to point out that although the gain and loss entropies show qualitatively similar behavior, the trajectories traced out by the instantaneously normalized, reduced density matrices $\tilde{\rho}_{\text {Gain }}(t)$ and $\tilde{\rho}_{\text {Loss }}(t)$ in the Bloch ball are distinctly different (Fig. 5). The trajectory of the gain-sector density matrix is weighted towards the northern hemisphere, representing the largest amplifying mode, whereas the loss-sector density matrix trajectory is less heavily weighted. These differences lead to the slightly different behaviors of $S_{\text {Gain }}$ and $S_{\text {Loss }}$.

In this Rapid Communication, we realize a four-level system dynamics under a non-Hermitian Hamiltonian in either $\mathcal{P} \mathcal{T}$-symmetric unbroken, broken, or at the exceptional point with single photons and a cascaded interferometric setup. We realize $4 \times 4$ nonunitary evolution operations with six BDs and use another one for state preparation. Two different measurements-projective measurement and the quantum state tomography of a four-level system - are carried out at the output. In contrast, the setup in Ref. [34] is much simpler; a two-level system dynamics under a non-Hermitian Hamiltonian is realized with two BDs, and only a single-qubit state tomography is carried out to reconstruct the final state. Our experimental method to implement a nonunitary, loss time evolution operator is scalable, and therefore can be used to simulate higher-dimensional $\mathcal{P} \mathcal{T}$-symmetric systems in the future.

\section{DISCUSSION}

In this section we briefly present the analytical derivation for the entropy of the $\mathcal{P} \mathcal{T}$-symmetric system. If we start with a pure state, it remains pure under the coherent, nonunitary evolution that is generated by a $\mathcal{P} \mathcal{T}$-symmetric, non-Hermitian Hamiltonian. Therefore, the entropy of such a state continues to remain zero. If the initial state is mixed, i.e., $\rho(0)=\sum_{i} \alpha_{i}\left|v_{i}\right\rangle\left\langle v_{i}\right|$, we can express the orthonormal vectors $\left|v_{i}\right\rangle=\sum_{k} \beta_{i k}\left|\zeta_{k}\right\rangle$ in terms of the nonorthogonal right eigenvectors $\left|\zeta_{k}\right\rangle$ of $H_{\mathcal{P} \mathcal{T}}$. The initial state thus can be rewritten as

$$
\rho(0)=\sum_{k, j, i} \alpha_{i} \beta_{i k} \beta_{i j}^{*}\left|\zeta_{k}\right\rangle\left\langle\zeta_{j}\right|
$$

The final state is then given by

$$
\rho(t)=\sum_{k, i} \alpha_{i}\left|\beta_{i k}\right|^{2}\left|\zeta_{k}\right\rangle\left\langle\zeta_{k}\left|+\sum_{k \neq j, i} \alpha_{i} \beta_{i k} \beta_{i j}^{*} e^{-i\left(\lambda_{k}-\lambda_{j}\right) t}\right| \zeta_{k}\right\rangle\left\langle\zeta_{j}\right|
$$

We further express the right eigenvectors of $H_{\mathcal{P} \mathcal{T}}$ in terms of the orthonormal eigenvectors of the instantaneous density matrix $\rho(t)$ as $\left|\zeta_{k}\right\rangle=\sum_{l} \kappa_{k l}\left|\varphi_{l}\right\rangle$. It allows us to obtain the time-dependent occupation eigenvalues $p_{k}(t)=\left\langle\varphi_{l}|\rho(t)| \varphi_{l}\right\rangle$ as

$$
p_{l}=\sum_{k, i, l} \alpha_{i}\left|\beta_{i k}\right|^{2}\left|\kappa_{k l}\right|^{2}+\sum_{k \neq j, i, l} \alpha_{i} \beta_{i k} \beta_{i j}^{*} \kappa_{k l} \kappa_{j l}^{*} e^{-i\left(\lambda_{k}-\lambda_{j}\right) t} .
$$

In the Hermitian limit, the eigenvectors of $H_{\mathcal{P}}$ are orthonormal, and the time evolution acts as the rotation of coordinates. Therefore the eigenstates $\left|\varphi_{i}\right\rangle$ are unchanged and the entropy remains a constant of motion. In the 
non-Hermitian case, $\left\{\left|\zeta_{k}\right\rangle\right\}$ are not orthonormal, and the timedependent entropy is then given by

$$
S(t)=-\sum_{l} \tilde{p}_{l} \log _{2} \tilde{p}_{l},
$$

where the fractional occupations are given by $\tilde{p}_{l}(t)=$ $p_{l}(t) / \sum_{k} p_{k}(t)$. The entropy of the time-evolved state oscillates periodically in the $\mathcal{P} \mathcal{T}$-symmetric unbroken region. At the EP4, $p_{l}(t)$ grow algebraically with time as $t^{6}$. By writing $p_{l}=\lambda_{l} t^{6}+\mu_{l}$ where $\lambda_{l}$ and $\mu_{l}$ are constant, it is straightforward to see that the entropy approaches a steady-state value polynomially with time. In contrast, in the $\mathcal{P} \mathcal{T}$-broken region, $p_{l}(t)$ grow exponentially with time, leading to a steady-state value for the entropy that is approaches in an exponential manner.

\section{SUMMARY}

Higher-dimensional $\mathcal{P} \mathcal{T}$ systems, which can be treated as composites of two or more minimal, non-Hermitian, quantum systems, provide a starting point for interacting quantum models with $\mathcal{P} \mathcal{T}$-symmetry and EP degeneracies. In this work, we experimentally simulate and observe the quantum information dynamics in a four-dimensional system with EP4. We show that the subsystem-entropy behavior for gain or loss subsystems can be either qualitatively different from or similar to the dynamics for the total entropy of the four-dimensional system. Our work is an experimental demonstration of critical phenomena in four-dimensional $\mathcal{P} \mathcal{T}$-symmetric quantum dynamics, and shows the versatility of the single-photon interferometric network platform for simulating interacting, non-Hermitian, quantum systems.

\section{ACKNOWLEDGMENTS}

The authors are thankful for support from the National Natural Science Foundation of China (Grants No. 11674056, No. 11974331, and No. U1930402), the National Natural Science Foundation of Jiangsu Province (Grant No. BK20190577), the Fundamental Research Funds for the Central Universities (JUSRP11947), the National Key R\&D Program (Grants No. 2016YFA0301700 and No. 2017YFA0304100), and NSF DMR-1054020.
[1] C. M. Bender, D. C. Brody, and H. F. Jones, Phys. Rev. Lett. 89, 270401 (2002).

[2] A. Mostafazadeh, J. Math. Phys. 43, 205 (2002).

[3] A. Mostafazadeh, Int. J. Geom. Methods Mod. Phys. 7, 1191 (2010).

[4] J. Carolan, C. Harrold, C. Sparrow, E. Martín-López, N. J. Russell, J. W. Silverstone, P. J. Shadbolt, N. Matsuda, M. Oguma, M. Itoh, G. D. Marshall, M. G. Thompson, J. C. F. Matthews, T. Hashimoto, J. L. O. Brien, and A. Laing, Science 349, 711 (2015).

[5] Y. N. Joglekar, C. Thompson, D. D. Scott, and G. Vemuri, Eur. Phys. J.: Appl. Phys. 63, 30001 (2013).

[6] C. E. Rüter, K. G. Makris, R. El-Ganainy, D. N. Christodoulides, M. Segev, and D. Kip, Nat. Phys. 6, 192 (2010).

[7] A. Regensburger, C. Bersch, M.-A. Miri, G. Onishchukov, D. N. Christodoulides, and U. Peschel, Nature (London) 488, 167 (2012).

[8] L. Feng, Z. J. Wong, R.-M. Ma, Y. Wang, and X. Zhang, Science 346, 972 (2014).

[9] B. Peng, Ş. Kaya Özdemir, F. Lei, F. Monifi, M. Gianfreda, G. L. Long, S. Fan, F. Nori, C. M. Bender, and L. Yang, Nat. Phys. 10, 394 (2014).

[10] L. Chang, X. Jiang, S. Hua, C. Yang, J. Wen, L. Jiang, G. Li, G. Wang, and M. Xiao, Nat. Photonics 8, 524 (2014).

[11] M. Brandstetter, M. Liertzer, C. Deutsch, P. Klang, J. Schöberl, H. E. Türeci, G. Strasser, K. Unterrainer, and S. Rotter, Nat. Commun. 5, 4034 (2014).

[12] S. Weimann, M. Kremer, Y. Plotnik, Y. Lumer, S. Nolte, K. G. Makris, M. Segev, M. C. Rechtsman, and A. Szameit, Nat. Mater. 16, 433 (2016).

[13] S. Assawaworrarit, X. Yu, and S. Fan, Nature (London) 546, 387 (2017)
[14] L. Feng, R. El-Ganainy, and L. Ge, Nat. Photonics 11, 752 (2017).

[15] H. Hodaei, A. U. Hassan, S. Wittek, H. Garcia-Gracia, R. ElGanainy, D. N. Christodoulides, and M. Khajavikhan, Nature (London) 548, 187 (2017).

[16] C. M. Bender and S. Boettcher, Phys. Rev. Lett. 80, 5243 (1998).

[17] T. Kato, Perturbation Theory for Linear Operators, Classics in Mathematics Vol. 132 (Springer, Berlin, 1980).

[18] W. D. Heiss, J. Phys. A 45, 444016 (2012).

[19] Ş. K. Özdemir, S. Rotter, F. Nori, and L. Yang, Nat. Mater. 18 783 (2019).

[20] B. Peng, Ş. K. Özdemir, S. Rotter, H. Yilmaz, M. Liertzer, F. Monifi, C. M. Bender, F. Nori, and L. Yang, Science 346, 328 (2014).

[21] J. Zhang, B. Peng, Ş. K. Özdemir, K. Pichler, D. O. Krimer, G. Zhao, F. Nori, Y. xi Liu, S. Rotter, and L. Yang, Nat. Photonics 12, 479 (2018).

[22] F. Minganti, A. Miranowicz, R. W. Chhajlany, and F. Nori, Phys. Rev. A 100, 062131 (2019).

[23] F. Minganti, A. Miranowicz, R. W. Chhajlany, I. I. Arkhipov, and F. Nori, Phys. Rev. A 101, 062112 (2020).

[24] C.-Y. Ju, A. Miranowicz, G.-Y. Chen, and F. Nori, Phys. Rev. A 100, 062118 (2019).

[25] Y. Han, X.-Q. Luo, T.-F. Li, W. Zhang, S.-P. Wang, J. S. Tsai, F. Nori, and J. Q. You, Phys. Rev. Appl. 11, 014053 (2019).

[26] A. Miranowicz, Ș. K. Özdemir, J. Bajer, G. Yusa, N. Imoto, Y. Hirayama, and F. Nori, Phys. Rev. B 92, 075312 (2015).

[27] Z. Xiao, H. Li, T. Kottos, and A. Alù, Phys. Rev. Lett. 123, 213901 (2019).

[28] H. Jing, Ş. K. Özdemir, H. Lü, and F. Nori, Sci. Rep. 7, 3386 (2017).

[29] C. M. Caves, Phys. Rev. D 26, 1817 (1982). 
[30] S. Scheel and A. Szameit, Europhys. Lett. 122, 34001 (2018).

[31] L. Xiao, X. Zhan, Z. H. Bian, K. K. Wang, X. Zhang, X. P. Wang, J. Li, K. Mochizuki, D. Kim, N. Kawakami, W. Yi, H. Obuse, B. C. Sanders, and P. Xue, Nat. Phys. 13, 1117 (2017).

[32] K. Wang, X. Qiu, L. Xiao, X. Zhan, Z. Bian, W. Yi, and P. Xue, Phys. Rev. Lett. 122, 020501 (2019).

[33] K. Wang, X. Qiu, L. Xiao, X. Zhan, Z. Bian, B. C. Sanders, W. Yi, and P. Xue, Nat. Commun. 10, 2293 (2019).

[34] L. Xiao, K. Wang, X. Zhan, Z. Bian, K. Kawabata, M. Ueda, W. Yi, and P. Xue, Phys. Rev. Lett. 123, 230401 (2019).

[35] Z. Bian, L. Xiao, K. Wang, X. Zhan, F. A. Onanga, F. Ruzicka, W. Yi, Y. N. Joglekar, and P. Xue, Phys. Rev. Research 2, 022039 (2020).

[36] X. Zhan, L. Xiao, Z. Bian, K. Wang, X. Qiu, B. C. Sanders, W. Yi, and P. Xue, Phys. Rev. Lett. 119, 130501 (2017).

[37] L. Xiao, T. S. Deng, K. K. Wang, G. Y. Zhu, Z. Wang, W. Yi, and P. Xue, Nat. Phys. 16, 761 (2020).
[38] F. Klauck, L. Teuber, M. Ornigotti, M. Heinrich, S. Scheel, and A. Szameit, Nat. Photonics 13, 883 (2019).

[39] J. Li, A. K. Harter, J. Liu, L. de Melo, Y. N. Joglekar, and L. Luo, Nat. Commun. 10, 855 (2019).

[40] M. Naghiloo, M. Abbasi, Y. N. Joglekar, and K. W. Murch, Nat. Phys. 19, 1232 (2019).

[41] E. M. Graefe, U. Günther, H. J. Korsch, and A. E. Niederle, J. Phys. A 41, 255206 (2008).

[42] M. A. Quiroz-Juárez, A. Perez-Leija, K. Tschernig, B. M. Rodríguez-Lara, O. S. Magaña-Loaiza, K. Busch, Y. N. Joglekar, and R. de J. León-Montiel, Photonics Res. 7, 862 (2019).

[43] W. Chen, Şahin Kaya Özdemir, G. Zhao, J. Wiersig, and L. Yang, Nature (London) 548, 192 (2017).

[44] K. Kawabata, Y. Ashida, and M. Ueda, Phys. Rev. Lett. 119, 190401 (2017).

[45] Y. Wu, W. Liu, J. Geng, X. Song, X. Ye, C.-K. Duan, X. Rong, and J. Du, Science 364, 878 (2019). 